\title{
Development and in-vitro Evaluation of a Topical Drug Delivery System Containing Betamethazone Loaded Ethyl Cellulose Nanospheres
}

\author{
Kumaraswamy Santhi, Dhandapani Nagasamy Venkatesh*, \\ Sokkalingam Arumugam Dhanaraj, Shanmugasundaram \\ Sangeetha and Bhojraj Suresh \\ Dept of Pharmaceutics, J S S College of pharmacy, Ooty - 643 001. Tamil Nadu, India.
}

\begin{abstract}
Purpose: Lipid nanospheres are used for the passive targeting of cosmetic agents to skin, thereby achieving major benefits such as reduction of total dose and avoidance of systemic absorption. The present study was carried out to exploit the feasibility of using polymeric nanospheres as an alternative and cheaper carrier for targeting corticosteroids to the skin.

Methods: Nanospheres were prepared from ethyl cellulose by a modified method of desolvation and cross linking. The drug betamethazone was incorporated into nanospheres and the drug: polymer ratio was evaluated to determine the carrier capacity of the polymer. In vitro release studies of drug-loaded nanospheres were carried out by the centrifugal ultrafiltration method. The kinetics of release was determined and fitted to an empirical equation. The release of drug from drug-loaded nanospheres dispersing in a conventional cream was evaluated. A comparative in vitro diffusion study was carried out between a commercial brand of cream and the cream incorporating nanospheres.

Results: Formulation of nanospheres of betamethazone by a modified method produced discrete particles. Studies on drug:polymer ratio showed a linear relationship between drug concentration and percentage of loading. The in vitro release of drug-loaded nanospheres was found to be first order. The comparative in vitro diffusion study between the commercial cream and the formulated cream showed a marked reduction in release rate from nanospheres-bound cream.
\end{abstract}

Conclusion: Formulated topical cream containing nanospheres of betamethazone was found to be a potential dermal delivery system for sustaining the release of the drug.

Keywords: Nanospheres, desolvation and cross-linking method, ethyl cellulose, betamethazone, in-vitro diffusion studies.

*Corresponding Author. Tel: 91-423-2443393. Fax: 91-423-2442937.Email: dnvooty@sify.com 


\section{Introduction}

In recent years, there has been a considerable interest in the development of novel drug delivery systems in order to modify and control pharmacokinetic behavior of the drugs. By incorporation into a carrier system, it is possible to alter the therapeutic index and duration of activity of drugs ${ }^{1}$. Colloidal drug delivery system represents one of the frontiers of science, which offers numerous advantages such as improved efficiency, reduced toxicity and improved patient compliance over conventional dosage forms. Nanoparticles are colloidal drug delivery systems that offer site-specific as well as controlled drug delivery $^{2-4}$. Many of the conventional vehicles such as creams, ointments requires high concentration of active ingredients for effective therapy due to their low efficiency as delivery systems ${ }^{5}$. Over the past decade, particulate carriers such as liposomes and microspheres have been investigated for the delivery of drugs to specific skin compartments in order to control drug input, release, retention and action in/into the skin and also to reduce systemic absorption and consequently adverse reactions. In vitro and in vivo experiments proved particulate carriers to be worthwhile for increasing cutaneous bioavailability and decreasing systemic absorption of many drugs ${ }^{6}$. The topical bioavailability of 5-aminolevulinic acid was improved when loaded into liposomes ${ }^{7}$. Incorporation of corticosteroids in liposomes provided a higher concentration and efficient delivery of corticosteroids..$^{8-9}$ Hence, in the present study, an attempt has been made to formulate ethyl cellulose nanospheres containing betamethazone, which may provide prolonged drug delivery in the treatment of inflammation and also minimize side effects such as pruritis, hypertrichosis and erythema.

\section{Experimental}

\section{Materials}

Betamethazone (Glaxo SmithKline Pharmaceuticals Ltd), Ethyl cellulose (Sigma), Tripolyidine phosphate (Sigma Chemicals), dialysis membrane (Sigma Chemicals), sodium hydroxide (LR grade), linseed oil (commercial grade), Tween 80 (LR grade, SD Fine Chemicals), toluene and potassium dihydrogen phosphate (LR grade, S.D Fine Chemicals) and ethanol (AR grade) were used.

\section{Methods}

Preparation of betamethazone nanospheres by desolvation and crosslinking method ${ }^{10}$ and study on drug : polymer ratio

An ethanolic solution $(50 \mathrm{ml})$ containing betamethazone $6.25 \mathrm{mg}$, ethyl cellulose $(1 \%$ $\mathrm{w} / \mathrm{v})$ and Tween $80(1 \% \mathrm{w} / \mathrm{v})$ was desolvated by stirring at $200 \mathrm{rpm}$ at $30 \pm 1{ }^{\circ} \mathrm{C}$ using distilled water. The extent of desolvation was monitored by visually by its turbid appearance and through optical microscopy. By trial and error method, it was determined that for every $50 \mathrm{ml}$ of ethanolic solutions of ethyl cellulose, a minimum of $12 \mathrm{ml}$ of water is required for desolvation. Tripolydine phosphate $(10 \mathrm{ml}$ of $15 \%)$ was added slowly to the suspension and stirred and continuously for $1 \mathrm{hr}$. The resultant turbid dispersion was dried at $30 \pm 1{ }^{\circ} \mathrm{C}$ on a glass plate. Ethyl cellulose nanospheres, along with the impurities, were obtained as a powder mass. Water $(5 \mathrm{ml})$ was added and centrifuged at $5000 \mathrm{rpm}$ for $10 \mathrm{~min}$. After washing with water, the nanospheres were collected in the supernatant layer as a thin ringlike structure, filtered and dried. Following this procedure, four other batches of ethyl cellulose nanospheres with varying concentrations of betamethazone - 12.5, 18.8, 25 and $50 \mathrm{mg}$ were prepared and coded batch B,C,D and E. For the determination of drug loading in each batch only the concentration of the drug was varied but other processing variables were kept constant.

Estimation of amount of drug incorporated into ethyl cellulose nanospheres

A quantity of drug-loaded nanospheres equivalent to $20 \mathrm{mg}$ of drug from each batch placed in a mortar, $10 \mathrm{ml}$ ethanol was added and triturated to crush the nanoparticles. This 
solution was then centrifuged and the drug content of the supernatant solution was analyzed by UV spectrophotometry at $267.6 \mathrm{~nm}$.

\section{Determination of particle size by transmission electron microscopy ${ }^{11}$}

The size and shape of the particles were also examined using TEM (Jeol EX 12, Japan). The specimens were prepared by dropping a mixture of phosphotungstic acid a dilute suspension of the nanospheres on EM grids (Formvar filmed, G200 mesh, Bio Rad, UK). Each grid was held for $20 \mathrm{~s}$ horizontally and then $45{ }^{\circ} \mathrm{C}$ to allow



Fig 1: Transmission electron micrograph of ethyl cellulose nanospheres containing betamethazone

excess fluid to drain. The specimens were air dried before examination with an electron microscope. The electron microscopic view of nanospheres containing betamethazone is shown in Fig 1.

In vitro release of drug loaded nanospheres before incorporating into cream base ${ }^{12-13}$

Table1: Formula for preparing creams

\begin{tabular}{cc}
\hline Ingredient & $\begin{array}{c}\text { For } 100 \mathrm{~g} \\
(\mathrm{w} / \mathrm{w})\end{array}$ \\
\hline Stearic acid & $18 \mathrm{~g}$ \\
Glycerine & $3 \mathrm{~g}$ \\
Lanolin & $2 \mathrm{~g}$ \\
Triethanolamine & $10 \mathrm{~g}$ \\
Preservative & $1 \mathrm{~g}$ \\
Perfume & $\mathrm{q} . \mathrm{s}$ \\
Water & $80 \mathrm{~mL}$ \\
\hline
\end{tabular}

A quantity of nanospheres equivalent to $20 \mathrm{mg}$ of drug from each batch was placed in a $250 \mathrm{ml}$ conical flask and to it $20 \mathrm{ml}$ ethanol and $80 \mathrm{ml}$ phosphate buffer $\mathrm{pH} 7.4$ were added. The flask was kept in a shaker incubator with the shaker oscillating at $20 / \mathrm{min}$ at $37{ }^{\circ} \mathrm{C}$. $5 \mathrm{ml}$ of drug release medium was withdrawn at various time intervals over a $24 \mathrm{~h}$ period. The samples were filtered and the drug content was analyzed by UV spectrophotometry at $267.6 \mathrm{~nm}$.

Invitro release of drug-loaded nanospheres after incorporation into cream

$10 \mathrm{mg}$ of drug-loaded nanospheres from each batch ' $D$ ' were placed in $250 \mathrm{ml}$ conical flask, and $10 \mathrm{ml}$ of ethanol and $90 \mathrm{ml}$ of phosphate buffer saline $(\mathrm{pH}$ 7.4) was added to it. The flask was kept in a shaker incubator oscillating at $20 / \mathrm{min}$ at $37 \pm 1^{\circ} \mathrm{C} .5 \mathrm{ml}$ of drug release medium was withdrawn at various time intervals over a $24 \mathrm{~h}$ period. These samples were vaccumfiltered through a membrane of $1 \mu \mathrm{m}$ pore size. The drug content was determined spectrophotometrically at $267.6 \mathrm{~nm}$.

Formulation of conventional cream and dispersion of drug loaded nanospheres in cream

Preparation of creams: Oil soluble ingredients from the formula were first dissolved in the oil phase and the water soluble ingredients were dissolved in the aqueous phase. Both the phases were prepared separately and heated to a temperature of $70^{\circ} \mathrm{C}$ separately. The oil phase was slowly incorporated into the aqueous phase by vigorous stirring until the formation of cream was smooth and uniform. The formulated cream was investigated for its physical parameters like consistency, homogenicity, spreadability, washability and extrudability. 


\section{Venkatesh et al}

Dispersion of drug loaded nanospheres into the cream base: A specific quantity of nanospheres from batch ' $D$ ' equivalent to $1 \mathrm{mg}$ of drug was incorporated into the selected cream base by simple mixing.

The medicated creams were then subjected to invitro diffusion studies. Three such sample batches were studied for their in vitro release characteristics.

In vitro diffusion studies of the cream containing dispersed nanospheres: Release of the drug, betamethazone, from the medicated creams was studied employing the permeation apparatus designed and described by Fites et $\mathrm{al}^{14}$. A glass cylinder with both ends open (10 cm high $3.7 \mathrm{~cm}$ outer diameter) was used as the permeation cell. A dialysis tubing (Sigma) soaked in a mixture glycerin and water (1:1) for $2 \mathrm{hr}$ before use was fixed to one end of the cylinder with the aid of an adhesive resulting in a permeation cell. The medicated cream (1 g) was placed in the cell (donor compartment) and the cell was immersed in a $250 \mathrm{ml}$ beaker containing drug-free diffusion medium, i.e., a mixture of $20 \mathrm{ml}$ of ethanol and $180 \mathrm{ml} 7.4 \mathrm{pH}$ phosphate buffer; which served as the receptor compartment. The cell was immersed to a depth of $1 \mathrm{~cm}$ below the surface of water in the receptor compartment. The medium in the receptor compartment was agitated using a magnetic stirrer at $37^{\circ} \mathrm{C} \pm 1^{\circ} \mathrm{C}$. A sample $(5 \mathrm{ml})$ of the receptor compartment fluid was taken at various intervals of time over a period of $24 \mathrm{~h}$ and analyzed for drug content at $267.6 \mathrm{~nm}$. The volume withdrawn each time was replaced with drug-free receptor fluid. The amount of drug released at various intervals of time was calculated and plotted against time as shown in Fig 2. This procedure was repeated for the conventional cream (Betnovate cream betamethazone $0.1 \% \mathrm{w} / \mathrm{w})$. The experiment was carried out in triplicate, and the mean cumulative percentage release from three batches was calculated.

\section{Results and Discussion}

The nanospheres prepared by desolvation and crosslinking method were found to have discrete

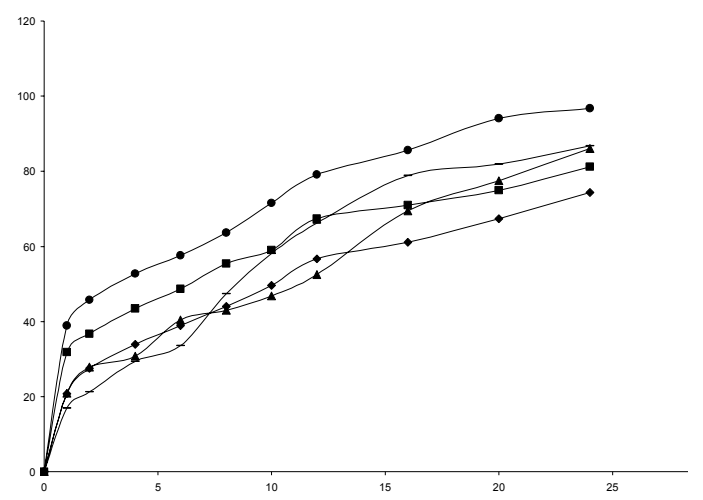

Figure 2: Invitro release profile of ethyl cellulose nanospheres containing various concentrations of betamethazone. Batch A ( $\mathbf{*})$, Batch B $(\diamond)$, Batch C $(\boldsymbol{\Delta})$, Batch D $(\bullet)$ and Batch $E(-)$.

particle size and shape within the size range of $703 \pm 11 \mathrm{~nm}$. The TEM of the drug-loaded nanospheres is shown Fig.1. There was a linear relationship between concentration of drug and percentage of loading up to a particular concentration $(25 \mathrm{mg} / \mathrm{mg}$ polymer). A decrease in drug loading was observed after that point due to the saturation capacity of polymer. The drug loading capacity of different batches are shown in Table 2. The drug loading was found to be $23.9 \pm 1.8 \%, 36.9 \pm 1.1 \%, 62.4 \pm 2.0 \%, 74.0 \pm 0.2 \%$ and $20.9 \pm 1.4 \%$ respectively for batches $A, B, C$, $\mathrm{D}$ and $\mathrm{E}$. In order to predict and correlate the release behavior of drugs from matrix, it is necessary to fit the data into a suitable model. The percentage of release from the drug-loaded nanospheres was in the range of 74 to $96 \%$. The in vitro release profile of betamethazone from all the formulated batches are shown in Fig 2 . Hence, the dissolution data were fitted according to the well known exponential equation, ${ }^{15}$ which is often used to describe drug release behavior from polymeric system.

$$
\mathrm{M}_{\mathrm{t}} / \mathrm{M}_{\infty}=\mathrm{Kt}^{\mathrm{n}}
$$




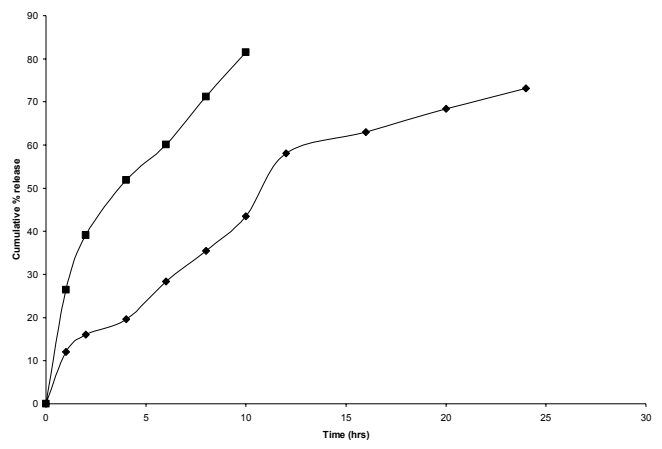

Figure 3: Comparative in vitro drug release from conventional cream ( $\square$ ) and nanosphereincorporated cream $(\diamond)$ by dialysis method

where, $M_{t} / M_{\infty}$ is the fractional release of the drug, ' $t$ ' is the release time, ' $k$ ' is a constant which incorporated the properties of the macromolecular polymeric system and ' $n$ ' is the release exponent indicative of the mechanism of release. The ' $n$ ' value used for analysis of the drug release mechanism from the nanospheres was determined from log $\left(M_{t} / M_{\infty}\right)$ Vs log $(t)$ plots. To calculate the release constant ' $k$ ', the logarithm of the remaining betamethazone in nanospheres is plotted versus time. The betamethazone nanospheres followed $1^{\text {st }}$ order release over the period. In Table 3 , the values of ' $k$ ', ' $n$ ' and ' $r$ ' for five different batches as reported, and the ' $n$ ' values were within 0.5122 to 0.5399 , and the ' $n$ ' value for the nanospheres bound cream was also 0.5272 . Thus the release mechanism is based on Fickian diffusion for both the nanospheres and the nanosphere bound cream. Among the different batches of drug loaded nanospheres, batch 'D' (prepared with 25 $\mathrm{mg}$ of drug/mg polymer) was found to possess a comparatively better and higher drug loading capacity. Therefore, batch ' $C$ ' was subjected to TEM analysis and size of drug loaded nanospheres were found to be slightly higher than the plain nanospheres. Among the different

Table 2: Betamethazone loading capacity of ethyl cellulose nanospheres

\begin{tabular}{|l|l|c|c|}
\hline S. No & Formulation code & $\begin{array}{c}\text { Drug concentration in } \\
\text { polymer } \\
(\mathrm{mg} / \mathrm{mg})\end{array}$ & $\begin{array}{c}\text { Drug loading capacity (\%) } \\
\pm \text { SD }\end{array}$ \\
\hline 1 & Batch 'A' & 6.25 & $23.95 \pm 1.8$ \\
\hline 2 & Batch 'B' & 12.5 & $36.95 \pm 1.1$ \\
\hline 3 & Batch 'C' & 18.8 & $62.44 \pm 2.0$ \\
\hline 4 & Batch 'D' & 25 & $73.99 \pm 0.2$ \\
\hline 5 & Batch 'E' & 50 & $20.91 \pm 1.4$ \\
\hline
\end{tabular}

Table 3: Invitro release kinetics of drug-loaded nanospheres

\begin{tabular}{|l|c|c|c|c|c|}
\hline Formulation & $\begin{array}{c}\text { Drug } \\
\text { code }\end{array}$ & \multicolumn{2}{|c|}{ First order plot } & \multicolumn{2}{c|}{ Koresmeyer and Peppas } \\
\cline { 3 - 6 } & $\begin{array}{c}\text { concentration } \\
\text { in polymer } \\
\text { (mg/mg) }\end{array}$ & $\mathrm{k}$ & $\mathrm{r}$ & $\mathrm{n}$ & $\mathrm{r}$ \\
\hline Batch 'A' & 6.25 & 0.0545 & 0.9947 & 0.5383 & 0.9225 \\
\hline Batch 'B' & 12.5 & 0.0469 & 0.9972 & 0.5389 & 0.9347 \\
\hline Batch 'C' & 18.8 & 0.0713 & 0.9789 & 0.5337 & 0.9304 \\
\hline Batch 'D' & 25 & 0.0794 & 0.9833 & 0.5123 & 0.9031 \\
\hline Batch 'E' & 50 & 0.0849 & 0.9924 & 0.5122 & 0.9065 \\
\hline $\begin{array}{l}\text { Nanosphere- } \\
\text { bound cream }\end{array}$ & & 0.0499 & 0.9993 & 0.5272 & 0.9955 \\
\hline
\end{tabular}


batches of nanospheres containing betamethazone, batch ' $D$ ' prepared with $25 \mathrm{mg}$ drug was incorporated into the cream. To compare the invitro release profile of betamethazone from nanospheres after incorporation into the cream with that of drug release from conventional cream comparative invitro diffusion study was carried out using phosphate buffer ${ }_{\mathrm{p}} \mathrm{H} 7.4$ containing $10 \%$ ethanol by dialysis method for $24 \mathrm{~h}$. The study revealed that the release of betamethazone from the conventional cream was found to be faster than from nanosphere bound cream. The cumulative release of drug from conventional cream at the $6^{\text {th }}$ hour was $57 \%$, while it was only $28 \%$ for the formulated cream. The $T_{50 \%}$ of the drug was found to be 3 and $11 \mathrm{~h}$, for the conventional and nanosphere-incorporated creams, respectively. The comparative invitro results obtained using the dialysis method are depicted in Fig 3. Thus, the formulated cream containing nanospheres exhibited sustained release over a period of 24 $\mathrm{h}$. Indicating that the dispersion of nanospheres in the cream base had a retarding effect on the release rate of the drug.

\section{Conclusion}

Formulation and evaluation of a topical drug delivery system containing betamethazone loaded nanospheres was found to be a potential and cost effective and sustained dermal delivery system in terms of particle size distribution, optimum drug loading capacity, and invitro diffusion characteristics. The system induced prolonged drug release and could, therefore, produce some benefits such as reduction in total dose, frequency of administration, and dose related systemic side effects.

\section{References}

1. Skiba M, Wouessidjewa D, Puisieux F, Duchene D and Gulic A. Characterization of amphiphilic $\beta$ cyclodextrin nanospheres. Int. J. Pharm. 1996; 142: $121-124$.

2. Gwen M Jantzen, Joseph R Robinson, Inc; Sustained and controlled release drug delivery system. Gilberts Banker, Ghristopher and J.Rhodes eds., Eds; Modern pharmaceutics, $3^{\text {rd }}$ Edition, Vol.72, Marcel Dekker, 1996, 596-597.
3. Ravikumar MNV, Pranita Singh and Dutta PK. Effect of swelling on chitosan -amine oxide gel in extended release of drug. Indian Drugs. 1999; 36(6): 393398.

4. Shobha Rani R, Hiremath and Hota A. Nanoparticles as drug delivery systems. Indian J. Pharm. Sci. 1999; 61(2): 69-75.

5. Sergio Nacht, Martin Katz, Inc; The microsponge: A novel topical programmable delivery system. David W. Sborne, Anton H. Amann., Eds., Topical Drug Delivery Formulations, Marcel Dekker. Vol. 42. New York, 1990; 299-325.

6. Alain Rolland. Particulate carriers in dermal and transdermal drug delivery. Marcel Dekker. Vol 64 1993: 367-421.

7. Maria Bernadete R Pierre, Antoinio C Tedesco, Juliana M Marchetti and M Vitoria LB Bently. Stratum corneum lipids liposomes for the topical delivery of 5-amniolevulinic acid in photodynamic therapy of skin cancer: Preparation and invitro permeation study. BMC, Dermatolog. 2001; 1:5.

8. Schmid $\mathrm{MH}$, Korting, HC. Therepeutic progress with topical liposome drugs for skin disease. Adv.Drug Deliv. Rev 1996; 18: 335-342.

9. Bently MVLB, Kddor ERM, Vianna RF, Collet JH. The influence of lecithin and urea on the in vitro permeation of hydrocortisone acetate through skin from hairless mouse. Int. J. Pharm. 1997; 146: 255-262.

10. Mukherji, G, Murthy RSR, and Miglani, BD. Preparation and evaluation of cellulose nanospheres containing 5-flourouracil. Int. J. Pharm. 1990; 65: 1-5.

11. Lin V, Coombes, AGA. Preparation of sub-100nm human serum albumin nanospheres using a ${ }_{\mathrm{p}} \mathrm{H}$ coacervation method. Journal of Drug Targeting. 1993; Vol 1: 237-243.

12. Smith, JG, Sihr, RF and Chalker, DK. Corticosteroid induced atrophy and telangiectasia. Archives of Dermatology. 1976; 112, 115.

13. Ravikumar, HR, Dhanaraj, SA, Rajeev Dube and Suresh, B. Distribution of etoposide-loaded hydrophilic albumin microspheres in mice. Drug Development and Industrial Pharmacy. 1996; 22(9\&10): 1005-1008.

14. Chowdary, KPR, and Kumar, PA. Formulation and evaluation of topical drug delivery systems for ciprofloxacin. Indian J. Pharm. Sci. 1996; 58(2): 47-50.

15. Koresmeyer RW, Gurny R, Doelkar E, Buri P and Peppas NA. Mechanisms of solute release from porous hydrophilic polymers. Int. .J. Pharm. 1983; 15: 23-25. 